\title{
An overview on carbon nanotubes
}

\begin{abstract}
Carbon nanotubes (CNTs) are third allotropic form of carbon-fullerenes which consist of graphite sheet rolled up into a cylindrical tube to form either single-wall (SWCNTs) or multi-wall (MWCNTs). Methods such as: Electrical arc discharge, Laser ablation and Chemical vapor deposition are used to synthesize CNTs. CNTs can be considered as the nano-pharmaceuticals of choice depending on their vast application in drug delivery, drug targeting and in various other fields such as diagnostic, tissue regeneration. To be integrated into the biological systems, CNTs can be chemically modified or functionalised with therapeutically active molecules by forming stable covalent bonds or supra-molecular assemblies based on non-covalent interactions. Owing to their high carrying capacity, biocompatibility, and specificity to cells, various cancer as well diseased cells have been explored with CNTs for evaluation of pharmacokinetic parameters, cell viability, cytotoxicty, and drug delivery in cells. CNTs can also be modified with positively charged bio-molecules such as ammonium group and cationic amino acid lysine, which lead to easy complex formation with genes of interest. CNT-based contrast agent may enhance molecular imaging by improving detection sensitivity and selectivity. The semi-conducting SWCNTs exhibits photoluminescence in the near-infrared (NIR) region. The emission ranges of 800-2000 nm cover the biological tissue transparency window, which is therefore suitable for the imaging of biological systems through the luminescence created by semi-conducting SWCNTs.
\end{abstract}

Keywords: carbon nanotubes, SWCNTs, MWCNTs, functionalization, CNTs applications
Volume 3 Issue 5 - 2017

\section{Mayur Porwal,Vaibhav Rastogi,Arvind Kumar IFTM University, India}

Correspondence: Mayur Porwal, Faculty of Pharmacy, IFTM University, Lodhipur-Rajput, Moradabad, U.P. 2441 02, India, Tel +919719261023, Email porwal_mayur1985@rediffmail.com

Received: July 25, 2017 | Published: August 07, 2017
Abbreviations: CNTs, carbon nanotubes; CVD, chemical vapor deposition; SDS, sodium dodecyl sulphate; PVP, polyvinylpyrrolidone; HIPCO, high-pressure co-conversion; HEK, human epidermal keratinocytes; NIR, near-infrared region

\section{Introduction}

After the discovery of third allotropic form of carbon - fullerene in 1991, Sumio Iijima ${ }^{1}$ identified a new structural form of this allotrope, the cylindrical fullerene and named them as carbon nanotubes (CNTs). ${ }^{1}$ CNTs are graphene sheets rolled into a seamless cylinder that can be open ended or capped, having a high aspect ratio with diameters as small as $1 \mathrm{~nm}$ and a length of several micrometers. ${ }^{2}$ Depending on the number of sheets rolled into concentric cylinders, there are two broad categories of CNTs, namely, single-walled carbon nanotubes (SWCNTs) and multi-walled carbon nanotubes (MWCNTs). SWCNTs are made up of single graphene layer wrapped into a hexagonal close-packed cylindrical structure whose diameter varies from $0.4-3.0 \mathrm{~nm}$ and length ranges from $20-1000 \mathrm{~nm}$ and are held together by van - der Waal forces, which makes them easily twistable and more pliable. ${ }^{3}$ SWCNTs are produced by the electric arc, laser ablation, chemical vapor deposition (CVD) and gas-phase catalytic processes (HiPco or High-Pressure Co-Conversion).

MWCNTs consist of several coaxial cylinders, each made of a single graphene sheet surrounding a hollow core. The outer diameter of MWCNTs ranges from 2 to $100 \mathrm{~nm}$, while the inner diameter is in the range of $1-3 \mathrm{~nm}$, and their length is 1 to several $\mu \mathrm{m}$. Electric $\operatorname{arc}^{4}$ and chemical vapor deposition (CVD) $)^{5}$ are the main techniques for their production. Owing to the $\mathrm{sp}^{2}$ hybridization in MWCNTs, a delocalized electron cloud along the wall is generated which is responsible for the $\pi-\pi$ interactions between adjacent cylindrical layers in MWCNTs resulting in a less flexible and more structural defects.

\section{Functionalization}

Despite of the advantages of CNTs in targeting various types of cells, various constraints have been made on the biological and biomedical applications of CNTs due to their lack of solubility in aqueous medium as well as their toxicity caused by the hydrophobic surface. These limitations of CNTs can be overcome by a process called Functionalization. ${ }^{6}$ This process of functionalization is also helpful in conjugating the therapeutic molecule or the ligands to the CNTs either on the surface or on the ends of CNTs so that to render them active against cancer cells and/or other diseased cells.

\section{Covalent functionalization}

The chemical functionalization of CNT is based on two main approaches

a. Esterification or amidation of oxidized tubes

b. Sidewall covalent attachment of functional groups.

Esterification or amidation of oxidized tubes: CNT can be oxidized, by substituting hydrophilic group such as $\mathrm{OH}, \mathrm{COOH}$ to CNTs. This treatment results in the opening of CNT end caps, generating carboxylic groups suitable for further derivatization. ${ }^{7}$ In addition, carboxylic functions are created where the defects of the nano-tube side walls are present.

Sidewall covalent attachment of functional groups: Addition of functional groups to the side walls of carbon nanotubes is frequently carried out by chemical reactions between functional organic molecu- 
les (amphotericin B, polyethylene glycol, etc.) and the SWCNTs surface by using reactive species such as nitrenes, carbenes and radicals. ${ }^{8}$

\section{Non-covalent functionalization}

The suspension of non-covalent functionalized CNT showed greater preservation of their aromatic structure and their electronic character as compared to pristine CNTs. This type of functionalization can be done by the addition of hydrophilic polymers, biopolymers and surfactants to the walls of CNTs through weak bonds. A series of anionic, cationic and non-ionic surfactants have been already proposed to disperse nanotubes in aqueous media. Sodium dodecyl sulfate (SDS) and benzylalkonium chloride are other good examples of surfactants that non-covalently aggregated to the nanotube side walls and facilitate the dissolution of carbon nanotubes in water. The adhesion between surfactants and nanotube walls becomes very strong due to the $\pi-\pi$ stacking interactions resulted from the attachment of aromatic groups of the amphiphile surfactant in the aromatic network of the nanotube side walls, as evidenced in the case of adhesion of $\mathrm{N}-$ succinimidyl-1-pyrenebutanoate. ${ }^{9}$

In the solubilization of the CNT, polymers represent a good alternative to surfactants although they do not have better dispersion efficiency. ${ }^{10}$ In this, process, hydrophilic polymer wraps around the tubes and thus modifies the solubility and conductivity properties of the CNTs. For example, polyvinylpyrrolidone (PVP), having polar sides along its long chain, assists the dissolution of PVP/SWCNT aggregates in polar solvents. Similarly Star et al. have substituted poly (metaphenylenevinylene) to suspend SWNT in organic solvents. ${ }^{11}$ Biopolymers can also be used for the functionalization of CNTs. Nucleic acids are certainly ideal candidates to form supramolecular complexes based on $\pi-\pi$ stacking between the aromatic bases and the CNT surface. Indeed, Zeng et al. ${ }^{12}$ have described an easy way to solubilize carbon nanotubes by simple sonication in the presence of a single-strand DNA. ${ }^{12,13}$

\section{Mechanism of CNTs penetration in to the cell}

The functionalized carbon nanotubes have an ability to cross cell membranes. The internalization of CNT can be tracked by labelling the CNT with fluorescent agent and then monitoring the uptake by using epifluorescence and confocal microscopy. The exact cellular uptake pathway of CNT is complex, but it is in general considered that there are two possible pathways for CNTs to cross the cellular membrane and enters the cell ${ }^{14}$ :

a. Endocytosis dependent pathway (which includes receptor and non-receptor mediated internalization)

b. Endocytosis independent pathway (which includes diffusion, membrane fusion and direct pore transport).

The process of internalization depends on several parameters such as the size, length, nature of functional groups (covalently linked or non-covalently linked to the CNT), hydrophobicity, surface chemistry and the cell culture medium.

\section{Limitations of carbon nanotubes}

Despite of the exciting prospects of CNTs in drug delivery, there are some factors which limit the applications of CNTs. Presence of impurities, non-uniformity in morphology and structure, large surface area (leads to protein opsonization), hydrophobicity, insolubility and tendency of CNTs to bundle together are some obstacles for their nano-medical applications. ${ }^{15}$

Another key obstacle is the toxicity of CNTs. The observed toxicity has been largely attributed to the structural similarity between CNTs and asbestos fibers; therefore the toxicological evaluation of CNTs has received much attention in recent years. Since graphite and carbon materials have been associated with increased dermatitis and keratosis, therefore in a study Shvedova et al., ${ }^{16}$ investigated the exposure of cultured human epidermal keratinocytes ( $\mathrm{HaCaT})$ to SWNTs which caused oxidative stress and loss of cell viability, indicating that dermal exposure to CNTs may lead to these altered skin conditions. ${ }^{16}$ In another study, Monteiro-Riviere et al. ${ }^{17}$ exposed Human epidermal keratinocytes (HEK) to MWCNTs and found that MWCNT induces the release of proinflammatory cytokine interlukin 8 from HEK which initiate an irritation response on target epithelial cells in time dependent manner. ${ }^{17}$ Pulmonary toxicity was also investigated in a study in which very high concentrations of SWCNTs were directly instilled into the lungs of the animals; it was found that exposure to SWCNTs lead to the development of granulomas in rodents..$^{18}$ Before the widespread utilization of CNTs in the medical science, it is important to note that the chronic toxicity of CNT must be experimentally studied and the appropriate safeguards must be taken against the possible interactions among the CNTs and biological systems.

\section{Applications of CNTS}

CNTs offer a wide range of application in medical sciences. These fields of CNT applications are as follows:
a. CNTs in Drug Delivery
b. CNTs in Gene Delivery
c. CNTs in Peptide Delivery
d. CNTs in Imaging and Diagnostics
e. CNTs in Tissue Regeneration

\section{Conclusion}

In summary, carbon nanotubes are among those carbon nano materials which possess large surface area with chemically tuneable functional groups. Owing to great physical and mechanical properties, CNT represents themselves as a potent drug carrier as well as a great imaging candidate in medicine. CNTs potential to undergo functionalization with the therapeutic or sensing moieties through a series of chemical reactions has made them promising biocompatible nano candidate for the diagnosis and targeted treatment of refractory diseases such as cancer, CNS disorder and infectious diseases and also in the field of tissue regeneration.

\section{Acknowledgements}

The authors express their sincere thanks to Prof. R.M. Dubey, Vice Chancellor, IFTM University, Moradabad, Uttar Pradesh, India, for his constant encouragement and support to carry out this work.

\section{Conflict of interest}

The Authors confirm that this article has no conflict of interest. 


\section{References}

1. Iijima S. Helical microtubules of graphitic carbon. Nature. 1991;354:5658.

2. Elhissi AM, Ahmed W, Hassan IU, et al. Carbon nanotubes in cancer therapy and drug delivery. J Drug Deliv. 2012;2012:837327.

3. Kostarelos K, Lacerda L, Partidos CD, et al. Carbon nanotube-mediated delivery of peptides and genes to cells: translating nanobiotechnology to therapeutics. J Drug Deliv Sci Technol. 2005;15(1):41-47.

4. Ebbesen TW, Ajayan PM. Large-scale synthesis of carbon nanotubes. Nature. 1992;358:220-222.

5. Rao CN, Govindaraj A. Carbon Nanotubes from Organometallic Precursors. Acc Chem Res. 2002;35(12):998-1007.

6. Gomez-Gualdrón DA, Burgos JC, Yu J, et al. Carbon nanotubes: engineering biomedical applications. Prog Mol Biol Transl Sci. 2011;104:175-245.

7. MA Hamon, J Chen, $\mathrm{H} \mathrm{Hu}$, et al. Dissolution of single-walled carbon nanotubes. Adv Mater. 1999;11(10):834-840.

8. Holzinger M, Vostrowsky O, Hirsch A, et al. Sidewall functionalization of carbon nanotubes. Angew Chem Int Ed Engl. 2001;40(21):4002-4005.

9. Villaverde A. Nanoparticles in translational science and medicine. London: Academic Press; 2011. 104: 648 p.

10. Minko T. Soluble polymer conjugates for drug delivery. Curr Drug Discov Technol. 2005;2(1):15-20.

11. Star A, Stoddart JF, Steuerman D, et al. Preparation and Properties of Polymer-Wrapped Single-Walled Carbon Nanotubes We would like to acknowledge the following agencies and foundations for supporting various aspects of this work: the polymer synthesis and spectroscopic characterization of the nanotube-polymer complex was funded by ONR; the chemical preparation and AFM analysis of these materials was supported by the NSF; device fabrication and charge-transport measurements were funded by DARPA and ONR; and the nonlinear microscopy experiments were supported by DARPA and the Keck Foundation. Angew Chem Int Ed Engl. 2001;40(9):1721-1725.
12. Zheng M, Jagota A, Strano MS, et al. Structure-based carbon nanotube sorting by sequence-dependent DNA assembly. Science. 2003;302(5650):1545-1548.

13. Zheng M, Jagota A, Semke ED, et al. DNA-assisted dispersion and separation of carbon nanotubes. Nat Mater. 2003;2(5):338-342.

14. Lee Y, Geckeler KE. Carbon nanotubes in the biological interphase: the relevance of noncovalence. Adv Mater. 2010;22(36):4076-4083.

15. Cheng HM, Hou PX, Liu C. Purification of carbon nanotubes. Carbon. 2008;46:2003-2025.

16. Shvedova AA, Castranova V, Kisin ER, et al. Exposure to carbon nanotube material: assessment of nanotube cytotoxicity using human keratinocyte cells. J Toxicol Environ Health A. 2003;66(20):1909-1926.

17. Monteiro-Riviere NA, Nemanich RJ, Inman AO, et al. Multi-walled carbon nanotube interactions with human epidermal keratinocytes. Toxicol Lett. 2005;155(3):377-384.

18. Lam CW, James JT, McCluskey R, et al. Pulmonary toxicity of singlewall carbon nanotubes in mice 7 and 90 days after intractracheal instillation. Toxicol Sci. 2004;77(1):126-134. 